\title{
Molecular Heterogeneity of the Vascular Endothelium Revealed by In Vivo Phage Display
}

\author{
Daniel Rajotte, ${ }^{*}$ Wadih Arap, ${ }^{\star}$ Martin Hagedorn, ${ }^{*}$ Erkki Koivunen, ${ }^{\ddagger}$ Renata Pasqualini, ${ }^{*}$ and Erkki Ruoslahti ${ }^{\star}$ \\ $*$ Research Center, The Burnham Institute, 10901 North Torrey Pines Road, La Jolla, California 92037; and ${ }^{\ddagger}$ Department of Biochemistry, \\ Biocenter 1B. Viikinkaari 5, PL56, FIN-00014, University of Helsinki, Helsinki, Finland
}

\begin{abstract}
Vascular beds are known to differ in structure and metabolic function, but less is known about their molecular diversity. We have studied organ-specific molecular differences of the endothelium in various tissues by using in vivo screening of peptide libraries expressed on the surface of a bacteriophage. We report here that targeting of a large number of tissues with this method yielded, in each case, phage that homed selectively to the targeted organ. Different peptide motifs were recovered from each of these tissues. The enrichment in homing to the target organs relative to an unselected phage was 3-35-fold. Peptide sequences that conferred selective phage homing to the vasculature of lung, skin, and pancreas were characterized in detail. Immunohistochemistry showed that the phage localized in the blood vessels of their target organ. When tested, the phage homing was blocked in the presence of the cognate peptide. By targeting several tissues and by showing that specific homing could be achieved in each case, we provide evidence that organ- and tissue-specific molecular heterogeneity of the vasculature is a general, perhaps even universal, phenomenon. Our results also show that these molecular differences can serve as molecular addresses. (J. Clin. Invest. 1998. 102:430-437.) Key words: blood vessels • vascular markers • endothelial cells organ targeting - peptide libraries
\end{abstract}

\section{Introduction}

Vascular endothelial cells play a crucial role in many physiological processes, including blood-tissue exchange, activation and migration of white blood cells, fibrinolysis, and coagulation (1). The vascular system is important in a variety of pathological conditions, including the main causes of morbidity and mortality-atherosclerosis and cancer $(2,3)$.

There are indications that the vascular beds in different parts of the body are morphologically and functionally different $(4,5)$. This is particularly true of the lymphoid tissues, where the high endothelium is composed of cells that express

Address correspondence to Erkki Ruoslahti, The Burnham Institute, 10901 N. Torrey Pines Road, La Jolla, CA 92037. Phone: 619-6463125; FAX: 619-646-3198; E-mail: ruoslahti@burnham-inst.org

Received for publication 6 February 1998 and accepted in revised form 21 May 1998.

J. Clin. Invest.

(C) The American Society for Clinical Investigation, Inc. 0021-9738/98/07/0430/08 \$2.00

Volume 102, Number 2, July 1998, 430-437

http://www.jci.org unique adhesion molecules for lymphocyte homing (6-8). Moreover, metastasis into preferred organs by certain tumors may depend on interactions between tumor cells and organspecific molecules in vascular beds. Thus, endothelial cell membrane vesicles have been found to bind preferentially those tumor cells that metastasize to the tissue of origin of the endothelial vesicles $(9,10)$.

Identification of organ-specific vascular markers has progressed slowly, at least partly because of difficulties in isolating pure populations of endothelial cells from tissues. Moreover, isolated and cultured cells may lose their tissue-specific traits upon culture $(5,11,12)$. Thus, the phenotype of endothelial cells is unstable and likely to change when the cells are removed from their microenvironment.

Phage display peptide libraries are commonly used to obtain defined peptide sequences interacting with a particular molecule. In this system, peptides in as many as $10^{9}$ permutations are expressed on the phage surface by fusion to one of the phage surface proteins and the desired peptides are selected on the basis of binding to the target molecule (13). The strength of this technology is its ability to identify interactive regions of proteins and other molecules without preexisting notions about the nature of the interaction. Phage libraries have been used to select for peptides that bind immobilized proteins (14-17), carbohydrates (18), and for peptides that bind to cultured cells (19).

Recently, we reported on a new approach to the use of phage libraries. We showed that peptides capable of tissuespecific homing could be identified by performing a selection for that trait in vivo (20). In that work, we isolated peptides capable of homing to two organs, the brain and the kidneys. The objective of the present study was to conduct a broad assessment of the organ-specific molecular diversity in endothelia. We have targeted seven different organs and obtained, in each case, phage that homed preferentially to the target organ. Detailed analysis of the homing to three of these organs revealed unique peptide sequences that mediated selective phage homing to the vasculature of these organs. As these tissues were selected to be different in size, location, function, and embryological derivation, our results suggest that a widespread system of endothelial specificities exists in tissues.

\section{Methods}

Phage peptide libraries. Fuse5 vector and K91kan bacterial strain were a gift from G. Smith (21). Peptide phage libraries were constructed as described previously (16). The libraries used in this study were: $\mathrm{X}_{7}, \mathrm{CX}_{6} \mathrm{C}, \mathrm{CX}_{7} \mathrm{C}, \mathrm{CX}_{3} \mathrm{CX}_{3} \mathrm{CX}_{3} \mathrm{C}$, and $\mathrm{X}_{2} \mathrm{CX}_{4} \mathrm{CX}(\mathrm{C}=$ cysteine; $\mathrm{X}=$ any amino acid). The titer of the various libraries was $\sim 10^{12}$ transducing units $/ \mathrm{ml}(\mathrm{TU} / \mathrm{ml}) .{ }^{1}$ Purification of phage particles and se-

1. Abbreviations used in this paper: GST, glutathione-S-transferase; TU, transducing units. 
quencing of phage single-stranded DNA were performed as described (16).

In vivo selection. 2-mo-old $\mathrm{Balb} / \mathrm{c}$ and $\mathrm{Balb} / \mathrm{c}$ nude mice were purchased from Harlan Sprague Dawley (San Diego, CA). In vivo phage selection was performed as described previously, with a few modifications $(20,22)$. Briefly, mice were first anesthetized with 0.017 $\mathrm{ml} / \mathrm{g}$ of Avertin and then injected intravenously (tail vein) with $10^{10}$ TU of phage peptide library. A major modification to the earlier procedure was that after $5 \mathrm{~min}$ of phage circulation in vivo, the mice were perfused through the heart with 5-10 ml of DME (Sigma Chemical Co., St. Louis, MO). Organs were then weighed, homogenized, and the phage were rescued by infection with K91kan bacteria. For the second and third rounds of in vivo selection, at least 200 clones were picked from the previous round and grown individually. Cultures were then pooled, phage particles purified, and $10^{9} \mathrm{TU}$ of this pool were injected into mice. Phage ssDNA of individual clones from the second and third round of selection was prepared and the insert sequenced. Phage with sequences appearing repeatedly in both rounds were retained, and the selected phage were then characterized further by injecting them individually.

For in vivo targeting of the skin we used Balb/c nude mice to avoid contamination of the tissue with hair. The mice were injected intravenously with phage and perfused as described above. Any contact of the skin with perfused blood was avoided. Skin was removed in large sections and placed on an ice-cold plate with the hypodermis facing up. The skin was then scraped with a scalpel, yielding primarily the hypodermis, and leaving behind the epidermis and part of the dermis. The scraped skin tissue was then processed for phage recovery as described above.

For in vivo targeting of the retina, 2-mo-old female Simonson Albino rats were anesthetized using $50 \mathrm{mg} / \mathrm{kg}$ body weight sodium-phenobarbital. While under deep anesthesia, the rats were injected with $10^{10} \mathrm{TU}$ of a library. The injection was administered into the left ventricle of the heart. After 2-5 min circulation time, the anterior chamber with cornea and lens were removed and the retina was peeled off the remaining posterior chamber. The tissue was homogenized in $1 \mathrm{ml}$ ice-cold DME containing $1 \mathrm{mM}$ PMSF, $20 \mu \mathrm{g} / \mathrm{ml}$ aprotinin, and $1 \mu \mathrm{g} / \mathrm{ml}$ leupeptin (all from Sigma Chemical Co.). The tissue was washed three times with $1 \mathrm{ml}$ DME and containing protease inhibitors. The phage were rescued by infecting bacteria and used for the subsequent round of selection. To assess homing of individual phage to retina, a phage that provides ampicillin-resistance, fdAMPLAY88 (23; a gift from Richard N. Perham, Cambridge University, Cambridge, UK), was used as an internal control. Rats were injected with an equal amount of the selected and fdAMPLAY88 phage, and homing to retina was evaluated by comparing the number of TU rescued from the retina on tetracycline (selected phage) and ampicillin plates (fdAMPLAY88).

Glutathione-S-transferase (GST)-fusion proteins. PCR amplification of the peptide-coding inserts was performed on phage ssDNA using the primers: AGGCTCGAGGATCCTCGGCCGACGGGGCT (sense) and AGGTCTAGAATTCGCCCCAGCGGCCCC (antisense). Annealing temperature for $\mathrm{PCR}$ was $53^{\circ} \mathrm{C}$. PCR products were ethanol precipitated and digested with BamHI and EcoRI. Digested fragments were inserted in-frame into the same sites of pGEX2TK vector (Pharmacia; Uppsala, Sweden). Large-scale preparations of GST-fusion proteins were produced and purified according to manufacturer's instructions (Pharmacia). The molecular weight and purity of the GST-fusion proteins were examined by SDSPAGE.

Immunohistochemistry. Phage proteins were detected in tissues by immunostaining as described $(20,22)$. Briefly, anesthetized mice were injected with $10^{9} \mathrm{TU}$ of phage and perfused as described above. The organs were surgically removed and then fixed in Bouin's solution (Sigma Chemical Co.). An antiserum against M13 phage (Pharmacia) was used for the staining, followed by a peroxidase-conjugated secondary antibody (Sigma Chemical Co.).

\section{Results}

Organ-selective phage isolated by in vivo screening. To identify phage that home selectively to individual organs, we injected phage libraries intravenously into mice and subsequently rescued the phage from these targets. We performed in vivo screens on lung, pancreas, skin, intestine, uterus, adrenal gland, and retina. All the screens yielded enrichment in phage homing to the target organ. The phage homing to lung, pancreas, and skin were selected for a detailed analysis.

Fig. 1 shows the enrichment profile obtained in three rounds of selection when targeting the lungs with a $\mathrm{CX}_{6} \mathrm{C}$ li-

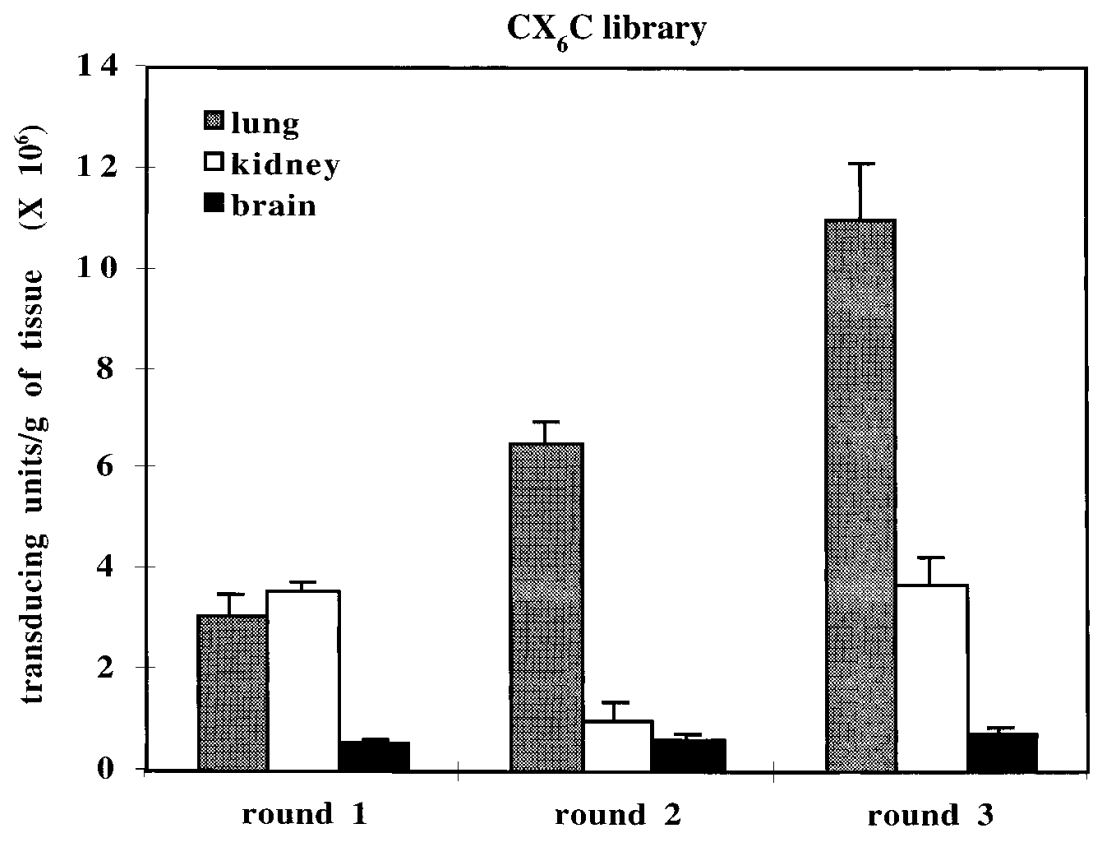

Figure 1. Selection of lung-targeting phage by in vivo screening of a phage-displayed peptide library. A $\mathrm{CX}_{6} \mathrm{C}$ library $\left(10^{10} \mathrm{TU}\right)$ was injected into the tail vein of mice. 5 min after the injection, the mice were perfused through the heart and phage were rescued from various organs. Phage recovered from the lungs was amplified and re-injected in two consecutive rounds. The number of TU recovered from lung, kidney, and brain tissue is shown. Error bars show standard deviation of the mean from triplicate platings. 

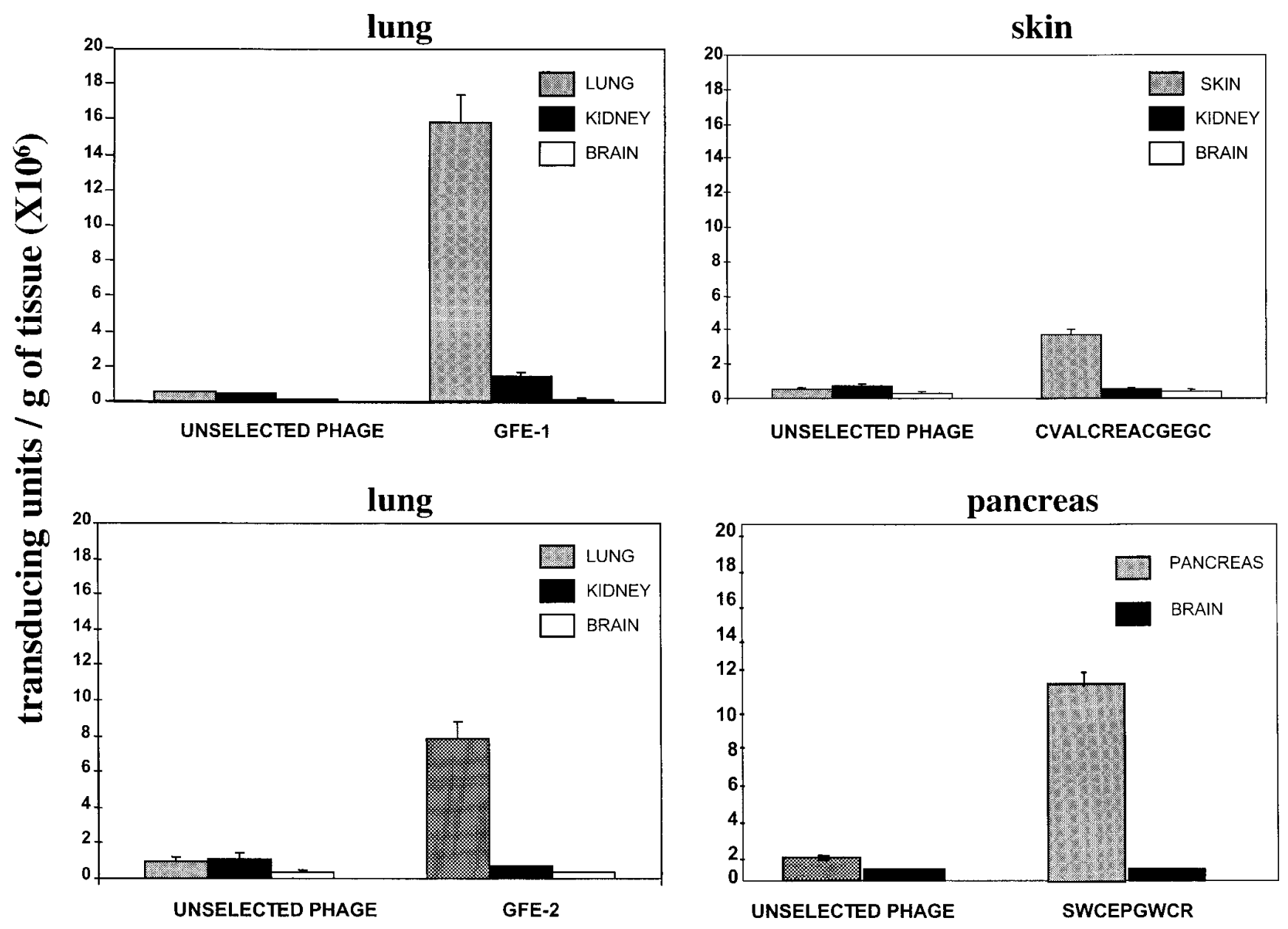

Figure 2. Specificity of organ-homing phage. Mice were injected with $10^{9} \mathrm{TU}$ of phage selected for organ homing or with unselected phage without insert. $5 \mathrm{~min}$ after the injection, the mice were perfused and phage were recovered from the indicated organs. The number of TU recovered is shown. Selective phage homing is shown for lung (GFE-1 and GFE-2), skin (CVALCREACGEGC), and pancreas (SWCEPGWCR) phage. Error bars show standard deviation of the mean from triplicate platings.

brary. The number of TU recovered from lung tissue markedly increased in the second and third rounds of selection, whereas the number of TU recovered from the kidney and the brain, which were used as controls, remained largely unaffected. In an earlier study, we observed an increase in phage homing to kidney and brain when targeting specifically these organs (20). The enrichment in the number of phage homing to lung upon selection was consistent and was also observed with libraries that had the general structures $\mathrm{CX}_{3} \mathrm{CX}_{3} \mathrm{CX}_{3} \mathrm{C}$ and $\mathrm{CX}_{7} \mathrm{C}$ (data not shown).

Phage that displayed the same or related peptide sequence appeared repeatedly in both second and third rounds of the lung screening. The lung-homing potential of these selected phage was then characterized further by injecting them individually. Four individual phage that homed to the lungs were identified. Two of these phage, isolated in independent screens, share the tripeptide GFE. The CGFECVRQCPERC (termed GFE-1) phage showed 35-fold and the CGFELETC (termed GFE-2) phage ninefold enrichment in homing to lung relative to an unselected phage (Fig. 2; Table I). No specific homing to kidney or brain could be detected. Two additional lung-homing phage from the $\mathrm{CX}_{6} \mathrm{C}$ library gave a sixfold
Table I. Targeting Sequences

\begin{tabular}{|c|c|c|}
\hline Organ/tissue & Displayed sequence & $\begin{array}{l}\text { Homing (Fold over } \\
\text { unselected phage) }\end{array}$ \\
\hline - & None & 1 \\
\hline Lung & CGFECVRQCPERC (GFE-1) & 35 \\
\hline Lung & 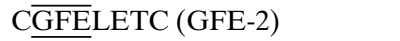 & 9 \\
\hline Lung & C $\overline{\overline{T L R}} \overline{D R N C}$ & 6 \\
\hline Lung & CIGEVEVC & 5 \\
\hline Skin & CVALCREACGEGC & 7 \\
\hline Pancreas & SWCEPGWCR & 6 \\
\hline Intestine & YSGKWGW & 10 \\
\hline Uterus & GLSGGRS & 3 \\
\hline Adrenal gland & LMLPRAD & 4 \\
\hline Retina & CSCFRDVCC & $3^{*}$ \\
\hline Retina & CRDVVSVIC & $2 *$ \\
\hline
\end{tabular}

The relative level of organ homing for each phage, compared to a phage without insert, is shown. Underlined sequences indicate common motifs displayed from either lung (double underlined) or retina (single underlined). *For retina, the level of phage homing was determined by using fdAMPLAY88 ampicilin-resistant phage as an internal control. 

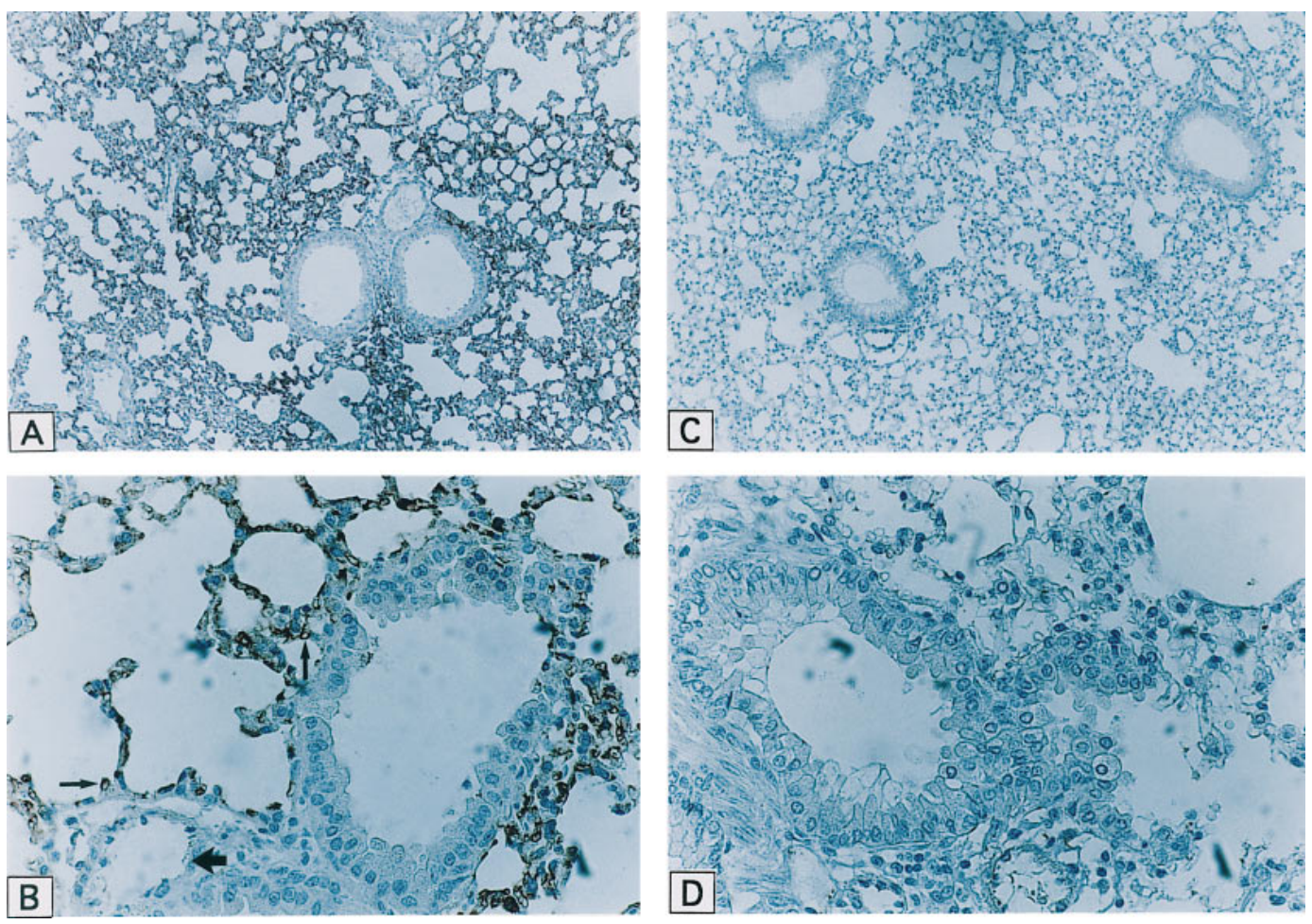

Figure 3. Immunohistochemical detection of phage homing to lung vasculature. GFE-1 ( $A$ and $B)$ or unselected $(C$ and $D$ ) phage were injected $\left(10^{9} \mathrm{TU}\right)$ into mice and allowed to circulate in vivo for $5 \mathrm{~min}$. After perfusion, tissue sections were prepared and subjected to immunoperoxidase staining to detect phage proteins. In $B$, small arrows are pointing to representative capillaries positive for phage staining. As indicated by the large arrow, larger blood vessels do not bind GFE-1 phage. Magnification: $A$ and $C: 100 \times ; B$ and $D: 400 \times$.
(CTLRDRNC phage) and fivefold (CIGEVEVC phage) enrichment over unselected phage (Table I). Interestingly, the EVE sequence in CIGEVEVC resembles the ELE tripeptide in GFE-2.

Targeting of skin with a $\mathrm{CX}_{3} \mathrm{CX}_{3} \mathrm{CX}_{3} \mathrm{C}$ library also yielded enriched phage homing (data not shown). The peptide sequence CVALCREACGEGC appeared repeatedly in the second and third rounds of the selection. When tested individually, this phage gave sevenfold selectivity for skin over unselected phage and over the background in brain and kidney (Fig. 2; Table I).

In a screen for the pancreas, the sequence SWCEPGWCR appeared multiple times in the second and third rounds of selection. Phage displaying this sequence showed approximately sixfold enrichment in homing to pancreas compared to unselected phage and the background in the brain was low (Fig. 2; Table I).

In vivo screens for intestine, uterus, adrenal gland, and retina yielded phage showing selective homing to these tissues. The peptide displayed by these phage and the corresponding enrichment are summarized in Table I.

Immunohistochemical staining for homing phage reveals vascular localization and organ specificity. Strong immunoperoxidase staining for phage was seen in lung vasculature 5 min after intravenous injection of the GFE-1 lung-homing phage (Fig. 3, $A$ and $B$ ). Alveolar capillaries were stained, whereas bronchiolar walls and some larger blood vessels were negative. No preference for any anatomical part of the lung was seen. Mice injected with unselected phage did not show staining in the lung (Fig. 3, $C$ and $D$ ). The GFE-2 phage was also detected by staining in the lung microvasculature, but the signal was weaker than for GFE-1 (data not shown). This weaker staining intensity of GFE-2 agrees with the phagecounting data, which also showed more lung binding by GFE-1 than GFE-2 (Fig. 2).

The skin-homing phage was detected on blood vessels of the hypodermis 5 min after intravenous injection; the dermis was not consistently stained (Fig. $4, A$ and $B$ ). Injecting an equal amount of unselected phage caused no staining in the blood vessels of the hypodermis or dermis (Fig. 4, $C$ and $D$ ). To determine whether the phage had access to the dermal blood vessels, we injected unselected phage at a 50-fold higher input than was used with the skin-homing phage and omitted the perfusion step. Although this procedure gave background staining in the deep dermal vessels, only occasional vessels of the dermis contained phage. This result suggests that circulating phage may not gain sufficient access to the dermal vessels to cause immunostaining. Thus, the apparent lack of dermal homing by the skin-homing phage may be due to poor access of the phage to these vessels rather than lack of a receptor.

The pancreas-homing SWCEPGWCR phage was found both in capillaries and larger blood vessels of the exocrine pancreas (Fig. $5 A$ ) and in the pancreatic islets (Fig. $5 \mathrm{~B}$ ). Again, an unselected phage caused no staining (Fig. 5, $C$ and $D$ ).

The lung- and skin-homing phage caused no staining in any of the other organs tested. These organs include kidney, brain, heart, muscle, lymph nodes, pancreas, intestine, and uterus (data not shown). The SWCEPGWCR pancreas-homing phage was an exception; it did give some staining in blood vessels within the uterus. Moreover, we recovered sixfold more SWCEPGWCR phage than unselected phage from the uterus after an intravenous injection (data not shown). Thus, this phage homes both to the pancreatic and uterine vasculature. All phage, including the unselected phage, caused staining in 

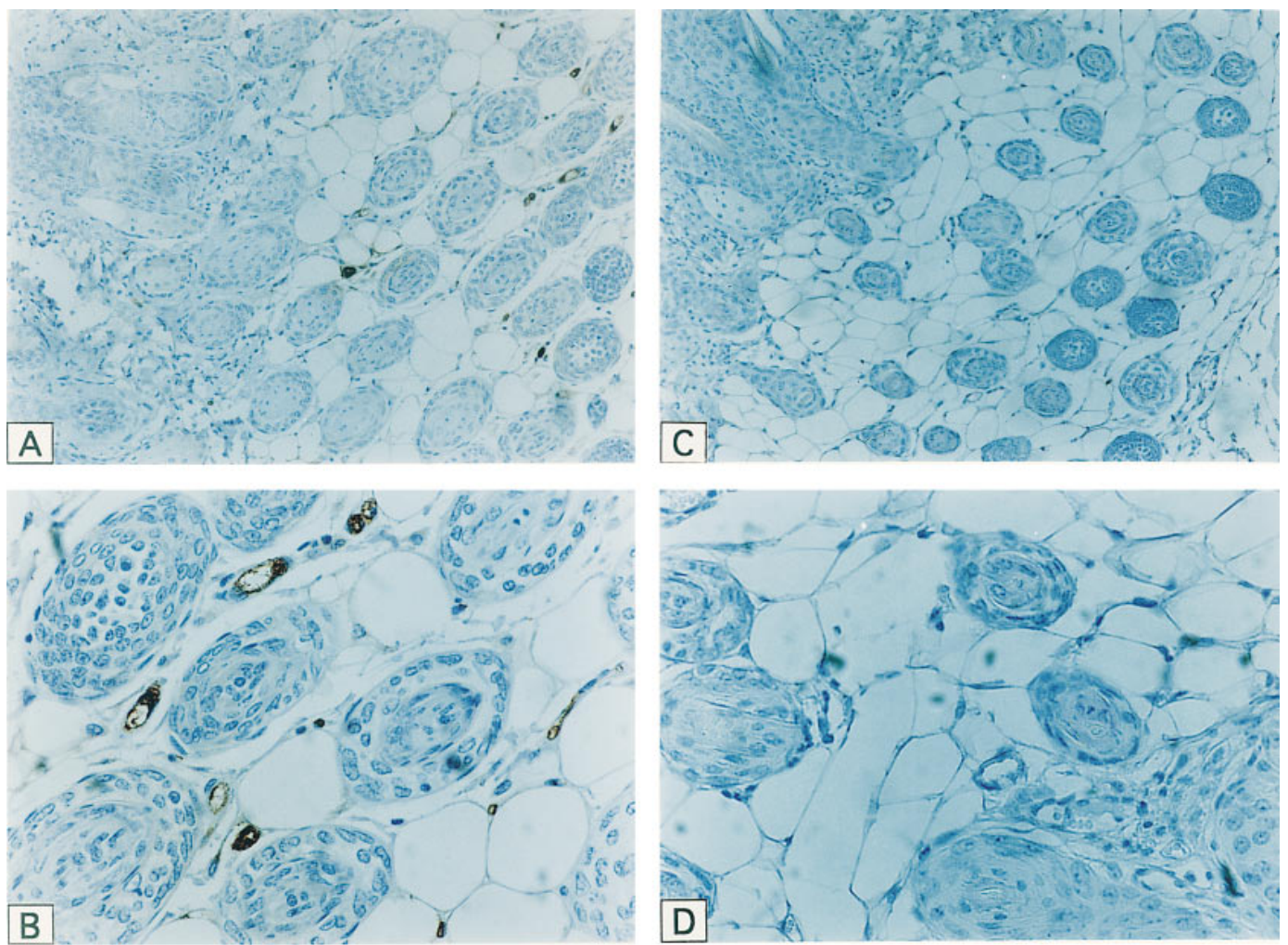

Figure 4. Immunohistochemical detection of phage homing to skin vasculature. Equal amounts of the CVALCREACGEGC phage ( $A$ and $B$ ), or unselected phage $(C$ and $D)$, were injected into the tail vein of individual mice and allowed to circulate for 5 min. After perfusion, sections were prepared and stained for the presence of phage proteins. Magnification: $A$ and $C$ : $100 \times ; B$ and $D: 400 \times$. the liver and spleen (data not shown). This is consistent with the previously described capture of circulating phage by the reticuloendothelial system $(20,22,24)$.

Cognate peptides compete for phage binding in vivo. The enrichment of certain sequences strongly suggested that the phage homing to target tissues depended on the peptide displayed by the phage, and not on some incidental mutant property of the phage. To confirm the role of the phage-displayed
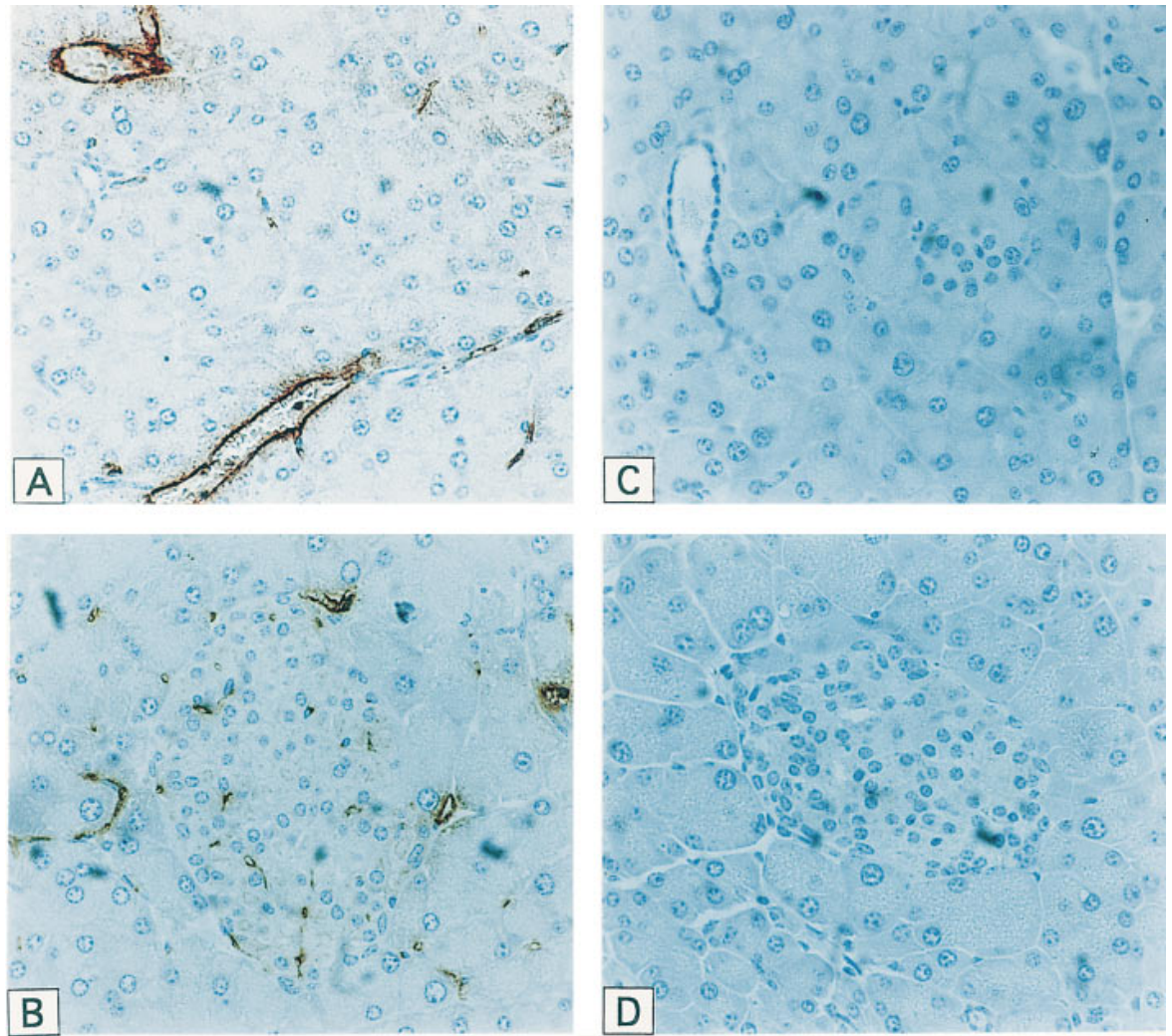

Figure 5. Immunohistochemical detection of phage homing to pancreas vasculature. Equal amounts of the CWCEPGWCR phage $(A$ and $B)$, or unselected phage $(C$ and $D)$, were injected into the tail vein of individual mice and allowed to circulate for 5 min. After perfusion, sections were prepared and stained for the presence of phage proteins. The exocrine pancreas $(A$ and $C$ ) and islets $(B$ and $D)$ are shown. Magnification: $160 \times$. 


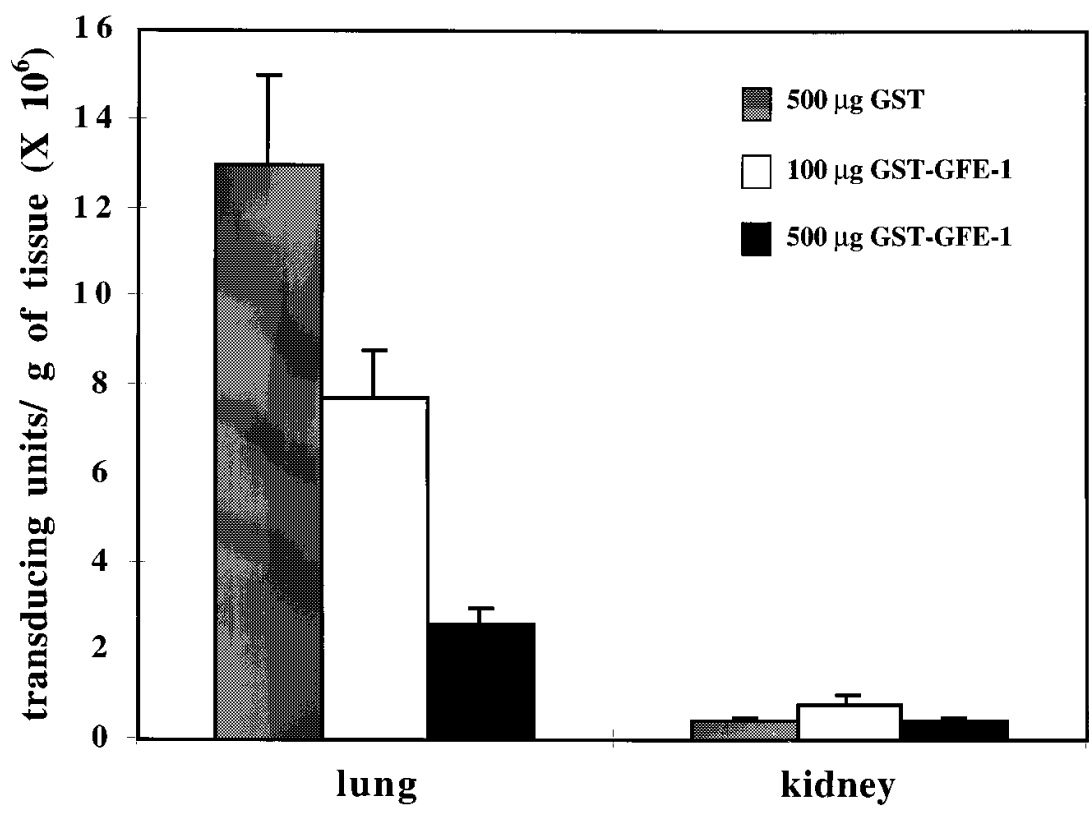

Figure 6. Effect of GST-GFE-1 fusion protein on GFE-1 phage homing in vivo. GST or GSTGFE-1, at the indicated amount, were injected into mice together with $10^{9} \mathrm{TU}$ of GFE-1 phage. 5 min after injection, phage were recovered from lung and kidney. Error bars indicate standard deviation of the mean from triplicate platings. sequences in the targeting to specific organs, we constructed GST-fusion proteins containing these sequences and used them as competitors for phage homing in vivo. A dose-dependent inhibition of the GFE-1 phage homing to lung tissue was observed when the GST-GFE-1 protein was co-injected with the phage; $500 \mu \mathrm{g}$ of GST-GFE-1 inhibited phage homing by $70 \%$ (Fig. 6). Co-injection of $500 \mu \mathrm{g}$ of GST protein did not affect lung homing of this phage. Moreover, GST-GFE-1 had no effect on the nonspecific recovery of phage from the kidney (Fig. 6). Lung homing of the GFE-2 phage was partially inhibited by the corresponding GST-fusion protein. At $500 \mu \mathrm{g}$, GST-GFE-2 inhibited the homing by $30 \%$ in several independent experiments. Higher concentrations did not improve the inhibition. The GST-GFE-1 protein at $500 \mu \mathrm{g}$ inhibited lung homing of the GFE- 2 phage by $60 \%$, but the same amount of the GST-GFE-2 protein had no effect on the homing of the GFE-1 phage. Possible reasons for the lack of GST-GFE-2 effect on the GFE-1 phage homing include that GFE-1 may have a higher affinity for a shared receptor, or that the GFE-2 fusion protein may aggregate at higher concentrations.

GST-CVALCREACGEGC inhibited the homing of CVALCREACGEGC phage to skin by $55 \%$. An equal amount of GST alone had no effect. The GST-CVALCREACGEGC protein also had no effect on the phage recovery to other organs such as lung and brain. There was no cross-inhibition among the lung-homing and skin-homing peptides; the GSTCVALCREACGEGC protein had no effect on the homing of the GFE phage; the GFE fusion proteins did not affect the CVALCREACGEGC phage (data not shown).

The homing of the SWCEPGWCR phage to pancreas was not inhibited reproducibly by the corresponding GST-fusion protein (data not shown). This lack of inhibitory activity could be due to a difference in the conformation of the peptide displayed by the two systems or to a higher binding affinity of the phage-displayed peptide than the GST-fusion protein for their ligand-multivalent display of a peptide can lead to an increase in binding affinity (16).

\section{Discussion}

We show here that vascular beds of a large number of tissues differ from one another, and we describe peptide sequencesidentified by in vivo screening of phage-displayed peptide libraries - that are capable of homing selectively to the vasculature of the lung, the skin, and the pancreas. Together with earlier results (20), these findings establish a system of tissuespecific individuality of vascular beds.

It may seem surprising that our method consistently yields tissue-specific homing peptides, rather than peptides that home nonselectively to any tissue. The phage libraries contain only few phage displaying of any given sequence. Injecting $10^{10}$ phage particles into a mouse means that $<100$ copies of any given phage are included. As this is a small number, any phage that binds to a ubiquitous endothelial marker will interact with multiple binding sites elsewhere than in the tissue of interest and will be rapidly depleted from the circulation. Thus, we believe that the selectivity is based on elimination of nonspecific phage binding at nontargeted sites. This would leave only those phage capable of binding selectively to the intended target tissue to be enriched there.

The potential of in vivo phage display for the identification of targeting sequences is obviously not exhausted, because we have tested relatively few types of libraries. Moreover, there would seem to be a variety of organ-specific markers targetable by our method, because it often yielded peptides with unrelated sequences that can home to the same vascular beds. However, as illustrated by the GFE (lung) and the RDV (retina) motifs, we may have targeted the same molecules repeatedly. These results indicate that a given vascular bed may have multiple specific markers, but that the number of such markers is finite.

We have, in this study, improved on our homing peptide selection by perfusing the mice after the injected phage had circulated. This reduced the nonspecific background of phage recovery. This was particularly useful in the targeting of the 
lungs, which we had previously found to yield high levels of background phage (20). In this study, we describe a protocol in which perfusion of the mice after phage circulation allows one to reduce that background. In addition, perfusion may increase the stringency of the screen, selecting for phage with higher affinities for the target. Moreover, we have recently developed an in vivo selection approach that includes noninfective phage as a competitor to thwart phage trapping in organs containing reticulo-endothelial tissue, such as liver and spleen. Thus, screening for peptides that home specifically to these tissues may also be possible (R. Pasqualini, E. Koivunen, and E. Ruoslahti, unpublished results).

It is interesting that the binding motif in the targeting peptides is often a tripeptide that appears in different sequence contexts. In in vivo screens for brain-homing phage the SRL tripeptide was found in several peptides (20). In the present work, we found a GFE motif in two lung-homing peptides and an RDV motif in two retina-homing peptides. In a different but related situation, the RGD motif is known to be important for integrin binding in distinct molecular contexts (25). Thus, many adhesive interactions seem to derive their specificity from tripeptide recognition motifs.

Our homing peptides bind to the vasculature in the target tissues. Earlier results have documented that for peptides homing to the brain, kidney, and several tumors $(20,22,26)$. Thus, the vasculature of many tissues carries a tissue-specific signature in the form of markers that can be detected by our phage display-based method. In some organs, such as pancreas and uterus, this signature may be shared by unrelated tissues. We have also obtained peptides that target the prostate with high selectivity (W. Arap, R. Pasqualini, and E. Ruoslahti unpublished results). Thus, including the brain and kidney homing reported earlier (20), the prostate and the seven organs reported here, the total number of organs targeted so far is ten. Given that we have been successful with every organ we have chosen to target, one can speculate that all tissues may "label" their vasculature with distinct markers.

There are other reports on tissue-selective molecular differences in endothelia (27). Possibly related to our findings on specific phage homing to the lung, dipeptidyl peptidase has been found to be expressed selectively in the lung endothelium and to serve as the receptor for a lung-metastasizing tumor cell line (28). Recently, Ghitescu et al. developed antibodies specific to the plasma membrane of rat lung microvascular endothelium (29). Two of the proteins detected by their antibodies show similar tissue distributions to our GFE phage. Direct comparison of the target molecules for the antibodies and our peptides is underway to resolve the question regarding their identity or dissimilarity. Moreover, surface protein differences have been detected by Dolichos biflorus agglutinin binding (30) and in situ glycoprotein labeling in endothelial surface proteins from liver, kidney, and brain (31). Finally, Aird et al. (32) recently showed that expression of the von Willebrand factor by endothelial cells can be regulated by organ-specific transcriptional pathways. These observations are in agreement with our results. However, the extent of tissue-specific molecular individuality of various vascular beds revealed by our data is unprecedented.

Our data also reveal molecular heterogeneity of the endothelium within a given organ. In the lungs, the GFE-1 and GFE-2 phage stained mainly capillaries rather than larger blood vessels. Interestingly, in the embryonic lung, capillaries do not arise as extensions of major blood vessels growing from the heart. Rather, the angioblasts within the lung give rise to capillaries that eventually become linked to extensions of the major blood vessels (33). Thus, our results may begin to delineate the molecular correlatives of such changes.

Our group has recently used in vivo selection of phage display libraries to isolate peptides that home specifically to tumor blood vessels (26). When coupled to the anticancer drug doxorubicin, these peptides enhanced the efficacy of the drug against human breast cancer xenografts in nude mice and also reduced its toxicity. Thus, our tissue-specific homing peptides may not only contribute to a better understanding of endothelial biology, but can also provide molecular tools for targeting individual vascular beds with diagnostic probes and therapeutic substances.

\section{Acknowledgments}

We thank K. Vuori (The Burnham Institute, La Jolla, CA) and G. Palade (University of California at San Diego, San Diego, CA) for comments on the manuscript, S. Krajewski for histological analysis, Brad Restel and the Institute's Animal Facility staff for their expert technical assistance.

This work was supported by grant CA 74238 to E. Ruoslahti, and the Cancer Center Support grant CA 30199. D. Rajotte is a Research Fellow of the National Cancer Institute of Canada supported with funds provided by the Terry Fox Run. W. Arap is the recipient of a CapCURE award. M. Hagedorn is a recipient of a fellowship from the Deutsche Forschungsgemeinschaft. E. Koivunen is supported by the Academy of Finland.

\section{References}

1. Fajardo, L.F. 1989. The complexity of endothelial cells. Am. J. Clin. Pathol. 92:241-250.

2. Gimbrone, M.A., M.I. Cybulsky, N. Kume, T. Collins, and N. Resnick. 1995. Vascular endothelium. An integrator of pathophysiological stimuli in atherogenesis. Ann. NY Acad. Sci. 748:122-131.

3. Hanahan, D., and J. Folkman. 1996. Patterns and emerging mechanisms of the angiogenic switch during tumorigenesis. Cell. 86:353-364.

4. Risau, W. 1995. Differentiation of endothelium. FASEB (Fed. Am. Soc. Exp. Biol.) J. 9:926-933.

5. Augustin, H.G., D.H. Kozian, and R.C. Johnson. 1994. Differentiation of endothelial cells: analysis of the constitutive and activated endothelial cell phenotypes. Bioessays. 16:901-906.

6. Streeter, P.R., E.L. Berg, B.T.N. Rouse, R.F. Bargatze, and E.C. Butcher. 1988. A tissue-specific endothelial cell molecule involved in lymphocyte homing. Nature. 331:41-46.

7. Lasky, L.A. 1992. Selectins: interpreters of cell-specific carbohydrate information during inflammation. Science. 258:964-969.

8. Salmi, M., and S. Jalkanen, 1997. How do lymphocytes know where to go: current concepts and enigmas of lymphocyte homing. Adv. Immunol. 64:139-218.

9. Johnson, R.C., H.G. Augustin-Voss, D. Zhu, and B.U. Pauli. 1991. Endothelial cell membrane vesicules in the study of organ preference of metastasis. Cancer Res. 51:394-399.

10. Auerbach, R., W.C. Lu, E. Pardon, F. Gumkowski, G. Kaminska, and M. Kaminski. 1987. Specificity of adhesion between murine tumor cells and capillary endothelium: an in vitro correlate of preferential metastasis in vivo. Cancer Res. 47:1492-1496.

11. Borsum, T., I. Hagen, T. Henriksen, and B. Carlander. 1982. Alterations in the protein composition and surface structure of human endothelial cells during growth in primary culture. Atherosclerosis. 44:367-378.

12. Bono, D.P., and C. Green. 1984. The adhesion of different cell types to cultured vascular endothelium: effects of culture density and age. Brit. J. Exp. Pathol. 65:145-154.

13. Burritt, J.B., C.W. Bond, K.W. Doss, and A.J. Jesaitis. 1996. Filamentous phage display of oligopeptide libraries. Anal. Biochem. 238:1-13.

14. Cwirla, S.E., E.A. Peters, R.W. Barrett, and W.J. Dower. 1990. Peptides on phage: a vast library of peptides for identifying ligands. Proc. Natl. Acad. Sci. USA. 87:6378-6382.

15. Wrighton, N.C., F.X. Farrell, R. Chang, A.K. Kashyap, F.P. Barbone, L.S. Mulcahy, D.L. Johnson, R.W. Barrett, L.K. Jolliffe, and W.J. Dower. 1996. 
Small peptides as potent mimetics of the protein hormone erythropoietin. Science. 273:458-463.

16. Koivunen, E., B. Wang, C.D. Dickinson, and E. Ruoslahti. 1994. Peptides in cell adhesion research. Methods Enzymol. 245:346-369.

17. Pasqualini, R., E. Koivunen, and E. Ruoslahti. 1995. A peptide isolated from phage display libraries is a structural and functional mimic of an RGDbinding site on integrins. J. Cell. Biol. 130:1189-1196.

18. Peletskaya, E.N., V.V. Glinsky, G.V. Glinsky, S.L. Deutscher, and T.P. Quinn. 1997. Characterization of peptides that bind the tumor-associated Thomsen-Friedenreich antigen selected from bacteriophage display libraries. $J$. Mol. Biol. 270: 374-384.

19. Barry, M.A., W.J. Dower, and S.A. Johnston. 1996. Towards cell-targeting gene therapy vectors: selection of cell-binding peptides from random peptide-presenting phage libraries. Nat. Med. 2:299-305.

20. Pasqualini, R., and E. Ruoslahti. 1996. Organ targeting in vivo using phage display peptide libraries. Nature. 380:364-366.

21. Smith, G.P., and J.K. Scott. 1993. Libraries of peptides and proteins displayed on filamentous phage. Methods Enzymol. 217:228-257.

22. Pasqualini, R., E. Koivunen, and E. Ruoslahti. 1997. AlphaV integrins as receptors for tumor targeting by circulating ligands. Nat. Biotechnol. 15:542-546.

23. Malik, P., and Pernham, R.N. 1997. Simultaneous display of different peptides on the surface of filamentous bacteriophage. Nucleic Acids Res. 25: 915-916.

24. Geter, M.R., M.E. Trigg, and C.R. Merril. 1973. Fate of bacteriophage lambda in non-immune germ-free mice. Nature. 246:221-223.

25. Ruoslahti, E. 1996. RGD and other recognition sequences for integrins.
Ann. Rev. Cell Dev. Biol. 12:697-715.

26. Arap, W., R. Pasqualini, and E. Ruoslahti. 1998. Cancer treatment by targeted drug delivery to tumor vasculature in a mouse model. Science. 279: $377-380$.

27. Belloni, P.N., and R.J. Tressler. 1989. Microvascular endothelial cell heterogeneity: interaction with leucocytes and tumor cells. Cancer Metastasis Rev. 8:353-389.

28. Johnson, R.C., D. Zhu, H.G. Augustin-Voss, and B.U. Pauli. 1993. Lung endothelial dipeptidyl peptidase IV is an adhesion molecule for lung-metastatic rat breast and prostate carcinoma cells. J. Cell Biol. 121:1423-1432.

29. Ghitescu, L.D., P. Crine, and B.S. Jacobson. 1997. Antibodies specific to the plasma membrane of rat lung microvascular endothelium. Exp. Cell Res. 232:47-55.

30. Ponder B.A.J., and M.M. Wilkinson. 1983. Organ-related differences in binding of Dolichos biflorus agglutinin to vascular endothelium. Dev. Biol. 96: 535-541.

31. Belloni, P.N., and G.L. Nicolson. 1988. Differential expression of cell surface glycoproteins on various organ-derived microvascular endothelia and endothelial cell cultures. J. Cell. Physiol. 136:398-410.

32. Aird, W.C., J.M. Edelberg, H. Weiler-Guettler, W.W. Simmons, T.W. Smith, and R.D. Rosenberg. 1997. Vascular bed-specific expression of an endothelial cell gene is programmed by the tissue microenvironment. J. Cell. Biol. 138:1117-1124.

33. Patanaud, L., F. Yassine, and F. Dieterlen-Lievre. 1989. Relationship between vasculogenesis, angiogenesis, and hemopoiesis during avian ontogeny. Development. 105:473-485. 